\title{
Diffuse Light Scattering Dynamics Under Conditions of Electromagnetically Induced Transparency
}

\author{
V. M. Datsyuk \\ I. M. Sokolov \\ D. V. Kupriyanov \\ M. D. Havey \\ Old Dominion University, mhavey@odu.edu
}

Follow this and additional works at: https://digitalcommons.odu.edu/physics_fac_pubs

Part of the Atomic, Molecular and Optical Physics Commons

\section{Repository Citation}

Datsyuk, V. M.; Sokolov, I. M.; Kupriyanov, D. V.; and Havey, M. D., "Diffuse Light Scattering Dynamics Under Conditions of Electromagnetically Induced Transparency" (2006). Physics Faculty Publications. 201.

https://digitalcommons.odu.edu/physics_fac_pubs/201

\section{Original Publication Citation}

Datsyuk, V. M., Sokolov, I. M., Kupriyanov, D. V., \& Havey, M. D. (2006). Diffuse light scattering dynamics under conditions of electromagnetically induced transparency. Physical Review A, 74(4), 043812. doi:10.1103/PhysRevA.74.043812 


\title{
Diffuse light scattering dynamics under conditions of electromagnetically induced transparency
}

\author{
V. M. Datsyuk, I. M. Sokolov, and D. V. Kupriyanov* \\ Department of Theoretical Physics, State Polytechnic University, 195251, St. Petersburg, Russia \\ M. D. Havey ${ }^{\dagger}$ \\ Department of Physics, Old Dominion University, Norfolk, Virginia 23529, USA
}

(Received 11 June 2006; published 13 October 2006)

\begin{abstract}
We show that under conditions typical of electromagnetically induced transparency (EIT) in an ultracold atomic sample in a magneto-optical trap, a significant portion of the incident probe pulse is transferred into Rayleigh and Raman scattering channels. The light scattered into the Rayleigh channel emerges from the sample with an EIT time delay. We show that a proper description of the probe light propagation in the sample should include, in the diffusion dynamics, a spin polariton generated by the two-photon EIT process. The results have important implications for studies of weak light localization and for manipulation of single and few photon states in ultracold atomic gases.
\end{abstract}

DOI: $10.1103 /$ PhysRevA.74.043812

PACS number(s): 42.50.Ct, 34.50.Rk, 34.80.Qb, 03.67.Mn

\section{INTRODUCTION}

External control of the electrodynamic response of atomic systems has been revolutionized by the merger of the ideas of coherent population trapping [1-4] with the techniques and concepts of ultracold atom physics [5]. The potential afforded by the combination was first demonstrated in the remarkable experiments of Hau et al. [6], in which a coherent light pulse was compressed in an ultracold gas of sodium atoms, the excitation having a very small group velocity, $\sim 17 \mathrm{~m} / \mathrm{s}$. Subsequent extensive theoretical and experimental research has shown that a combined atomic-photonic quasiparticle excitation is created in the ultracold medium and that the properties of the polariton can be dynamically manipulated through the external qualities of the light fields used to prepare and probe the medium [2-4,7]. Since then, studies have shown a broad range of physics associated with coherent manipulation of propagation of electromagnetic waves [8-15]. For example, Chanelière and coresearchers have recently shown, in a series of beautiful experiments [16], that it is possible to generate single-photon wave packets and to map them into polaritons in an ultracold sample of ${ }^{87} \mathrm{Rb}$ atoms. The single-photon wave packets could be regenerated after a controllable delay by judicious application of a control electromagnetic field. The physical processes associated with manipulation and storage of individual photon wave packets, and entanglement of the quantum states of the packets with a propagating one, are critical elements for quantum information protocols and quantum memory applications.

Among the essential elements needed for practical applications is quantitative understanding of coherence loss mechanisms in each stage of a quantum information protocol. In many studies of storage and retrieval of single and

\footnotetext{
*Electronic address: Kupr@DK11578.spb.edu

†Electronic address: mhavey@odu.edu
}

multiple quantum wave packets in ultracold gases, the initially created excitation undergoes decay as the length of the storage time is increased. In this paper we focus quantitatively on one mechanism which can lead to such a potential loss of fidelity and some of the surprising physics that results from these considerations. In particular, we consider the diffusely (multiply) scattered light that is necessarily generated as a result of optical excitation with temporally finite light pulses. In a general case, the associated finite spectral bandwidth produces diffusely scattered Rayleigh and Raman modes in the sample. These modes show quite different temporal behavior, in comparison with the coherent forward scattered probe light. In addition, because the Rayleigh scattered component maintains coherence with the incident probe beam, a new type of quasiparticle, a diffuse polariton, is created. Finally, we point out that the physics of light diffusion in a coherent medium consisting of a thermal (not ultracold) gas of $\mathrm{Rb}$ atoms has been investigated by Matsko et al. [14]. We point out that there have been a large number of studies of the fascinating physics of coherent population trapping; some representative papers are cited here [8-14]. A number of reviews have also appeared on the subjects of EIT and coherent population trapping, citing contemporary applications $[1,3,4,17,18]$.

In the remainder of this paper, we first consider in more detail why, in the context of the previous discussion, the diffuse scattering channels are important. This is followed by a description and discussion of the complex susceptibility for the case of a typical lambda configuration attainable on hyperfine resonance transitions in an ultracold gas of ${ }^{87} \mathrm{Rb}$ atoms [19]. The scattering channels are then considered, with particular attention paid to the Green's function for propagation in the inhomogeneous and optically anisotropic medium. We then present our results describing the temporal behavior of the forward scattered light and the diffusely scattered fluorescence. We finally show that the coherence of the multiply scattered Rayleigh mode can be detected through the appearance of the coherent backscattering effect for the weak probe beam. 


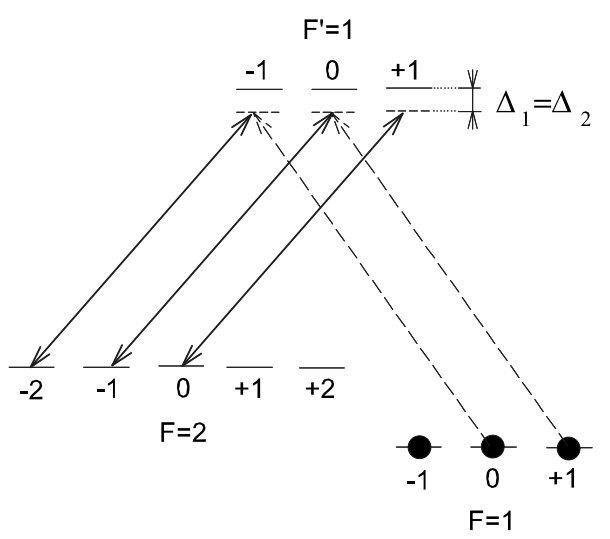

FIG. 1. An example of an excitation scheme for observation of the EIT effect in the system of hyperfine and Zeeman sublevels of the $D_{1}$ line of ${ }^{87} \mathrm{Rb}$. The coupling field is applied with right-handed circular polarization to the $F=2 \rightarrow F^{\prime}=1$ transition, and the probe mode in the orthogonal left-handed polarization excites the atoms on the $F=1 \rightarrow F^{\prime}=1$ transition. The EIT effect appears for equal detunings of the coupling and probe modes from atomic resonances: $\Delta_{1}=\Delta_{2}$.

\section{BASIC ASSUMPTIONS AND CALCULATION APPROACH}

\section{A. Why the scattering channels are important}

As an example of a system where electromagnetically induced transparency (EIT) resonance can be clearly observed, we consider the $\Lambda$-type configuration in the hyperfine manifold of the $D_{1}$ line of ${ }^{87} \mathrm{Rb}$ (see Fig. 1). A strong coupling field with right-handed circular polarization is applied between the $F=2$ hyperfine sublevel of the ground state and $F^{\prime}=1$ hyperfine component of the excited level. The atoms equally populate the relevant Zeeman states of the lower hyperfine sublevel $F=1$. In the figure, the detuning of the coupling (probe) laser from atomic resonance is defined as $\Delta_{1}$ $\left(\Delta_{2}\right)$. Such an atomic configuration is typical for EIT observation and can be fashioned in ultracold systems using, for example, magneto-optic or quasistatic dipole traps. Such configurations have also been widely studied in more traditional heated atomic gas cells; see for example, Refs. [12-14].

In the present paper, we assume that atoms are so deeply slowed by an atomic cooling process that we can neglect all effects associated with atomic motion. These are ideal conditions for EIT and the probe mode, being applied with any polarization to the $F=1 \rightarrow F^{\prime}=1$ hyperfine transition, would find respective $\Lambda$-type excitation channels, thus generating, as a result of the coherent Raman process, hyperfine coherence in the ground levels. To set our terminology, we refer in brief to Rayleigh and elastic Raman scattering processes as Rayleigh scattering. The terminology Raman scattering refers only to hyperfine inelastic Raman scattering. All time scales are measured relative to the inverse lifetime $\Gamma^{-1}$ of the ${ }^{87} \mathrm{Rb}$ resonance transition.

To reliably observe the conversion of the probe pulse into a polariton-type quasiparticle state, which, as expected, would forwardly propagate through the sample, some tempo- ral and spectral requirements should be fulfilled. There are two important temporal parameters for the process: the pulse duration $\tau_{p}$ and the delay time $\tau_{d}$ between the entrance and emergence of the probe light pulse. Apparently, for highfidelity conversion of the full incident light pulse into the polariton pulse, it is desirable that $\tau_{d}>\tau_{p}$. For the simplest $\Lambda$-type configuration the delay time can be estimated as

$$
\tau_{d}=\frac{L}{\bar{v}} \sim n_{0} \chi^{2} L \frac{\Gamma}{\Omega_{R}^{2}},
$$

where $L$ is the sample length, $\bar{v}$ is a group velocity for the probe mode at the resonance, $n_{0}$ is the density of atoms, $X$ is the wavelength divided by $2 \pi$ for the probe radiation, $\Gamma$ is the natural decay rate for the upper state, and $\Omega_{R}$ is the Rabi frequency for the coupling mode. The time $\tau_{p}$ can be estimated via the time-frequency uncertainty principle, i.e., as $\tau_{p} \sim 1 / \Delta_{p}$, where $\Delta_{p}$ is the spectral width of the pulse. The spectral width $\Delta_{p}$ is restricted by the condition that, at the relevant detuning from the EIT resonance, the optical thickness of the sample $b\left(\Delta_{2}\right)$ at $\Delta_{2}=\Delta_{p}$ would be small enough and the medium would be transparent. Then, in order of magnitude, the pulse duration $\tau_{p}$ is limited by the inequality

$$
\tau_{p}>\sqrt{n_{0} \chi^{2} L} \frac{\Gamma}{\Omega_{R}^{2}} .
$$

For an optically extended medium with $\sqrt{n_{0} \chi^{2} L} \gg 1$ the pulse duration can be made shorter than the delay time, but in reality such an optically dense sample is rather difficult to prepare in an experiment with ultracold atoms, where the parameter $n_{0} \chi^{2} L$ is typically close to ten or even less in order of magnitude.

The problem is more subtle if the EIT channel is adjusted for transport of a portion of "nonclassical" light. As a particular example, one can imagine a pulse of forwardly propagating squeezed light. Such states of light can be created with an intracavity optical parametric light source and its spectral properties can be controlled with the quality factor of the cavity. For such a light source the outgoing radiation can be properly described by the model proposed by Collett and Gardiner in Ref. [20] where the squeezed $\left(X_{1}\right)$ and antisqueezed $\left(X_{2}\right)$ quadrature components are described by the correlation functions with different relaxation times, which we respectively denote as $\tau_{1}$ and $\tau_{2}$. In principle, there exists an inequality between these correlation times $\tau_{2}>\tau_{1}$, which can be a strong constraint for high degrees of squeezing. For reliable transport of the squeezed light with preservation of its unique statistical properties it would be necessary that $\tau_{p}>\tau_{2}>\tau_{1}$. The variation of all these temporal parameters should be limited both by the longer estimation (2.1) and the shorter estimation (2.2). Apparently it would be more difficult to fulfill the EIT criteria for the squeezed light than for a pure coherent light source.

However, the atomic sample can be probed with a light pulse with shorter duration than is given by (2.2), and the EIT-mechanism will work even if a portion of the light pulse is transported via a nonforward scattering channel. For such an experimental situation the pulse duration $\tau_{p}$ can be limited by the time scale $\Gamma / \Omega_{R}^{2}$, given by the inverse spectral width 
of the transparency window in the local susceptibility of the medium. Then the input pulse of the probe light should be transformed into a polariton-type pulse in the diffuse mode, which now will fill the sample via a coherent diffusion (nonforward scattering) process.

\section{B. The macroscopic susceptibility and scattering tensors}

The dynamics of the macroscopic polarization, induced by a probe radiation pulse, is driven by the dynamical susceptibility of the medium. Referring to the excitation scheme shown in Fig. 1, under conditions of EIT resonance, the susceptibility of the medium for the probe mode entering the sample in a particular polarization is generated by two coherently interacting $\Lambda$-type channels. In the laboratory frame, with the $z$ axis directed along the coupling beam, the susceptibility tensor has a diagonal form in the basis of circular polarizations and can be written as the following sum

$$
\begin{aligned}
\chi_{q}^{q^{\prime}}\left(\mathbf{r}, \Delta_{2}\right)= & -\delta_{q}^{q^{\prime}} \sum_{n(m), m^{\prime}(m), m} \frac{1}{\hbar} \frac{\left.\mid \mathbf{d e}_{q}^{*}\right)\left._{n m}\right|^{2}}{\Delta_{2}+i \Gamma / 2} \rho_{m m}(\mathbf{r}) \\
& \times\left\{1-\frac{\left|V_{n m^{\prime}}\right|^{2}}{\Delta_{2}+i \Gamma / 2} \frac{1}{\Delta_{1}-\Delta_{2}+\Sigma_{n m^{\prime}}\left(\Delta_{2}\right)}\right\} .
\end{aligned}
$$

We use standard co-contravariant notation for the basis vectors of circular polarizations, see Ref. [21], which can be expressed by Cartesian basis vectors as $\mathbf{e}_{0}=\mathbf{e}_{z}, \mathbf{e}_{ \pm 1}$ $=\mp\left(\mathbf{e}_{x} \pm i \mathbf{e}_{y}\right) / \sqrt{2}[22]$.

The first line in (2.3) has an isotropic form and describes the local macroscopic susceptibility in the normal approach of linear electrodynamics. Here the squared transition dipole moments $\left(\mathbf{d e}_{q}^{*}\right)_{n m}$ between the lower $|m\rangle \equiv|F, m\rangle$ and upper $|n\rangle \equiv\left|F^{\prime}, n\right\rangle$ Zeeman sublevels are weighted with the population components of the atomic density matrix $\rho_{m m}(\mathbf{r})$ $=n_{0}(\mathbf{r}) /(2 F+1)$, where $n_{0}(\mathbf{r})$ is the local density of atoms at a spatial point $\mathbf{r}$. In a environment characteristic of ultracold and trapped atoms, the density distribution is typically inhomogeneous and the density matrix as well as the susceptibility tensor are spatially dependent. The frequency detuning $\Delta_{2}$ is the offset of the probe mode $\omega_{2}$ from the resonance $\Delta_{2}$ $=\omega_{2}-\omega_{F^{\prime} F}$ with $F=1, F^{\prime}=1$.

The second line in (2.3) reveals the contribution of the EIT effect. Here $V_{n m^{\prime}}$ are the transition matrix elements for the coupling mode between those quantum states $|n\rangle$ $\equiv\left|F^{\prime}, n\right\rangle$ and $\left|m^{\prime}\right\rangle \equiv\left|F, m^{\prime}\right\rangle$ which are subsequently chained with an initial state $|m\rangle$ via the respective $\Lambda$-type excitation channel. This is indicated in the sum by the dependence of the subscript indices on $m: m^{\prime}=m^{\prime}(m)$ and $n=n(m)$. The frequency detuning $\Delta_{1}$ is the offset of the coupling mode $\omega_{1}$ from the resonance $\Delta_{1}=\omega_{1}-\omega_{F^{\prime} F}$ with $F=2, F^{\prime}=1$. The pole in the denominator of Eq. (2.3) is shifted due to the AutlerTownes effect and the self-energy correction is given by

$$
\Sigma_{n m^{\prime}}\left(\Delta_{2}\right) \equiv \Delta_{n m^{\prime}}\left(\Delta_{2}\right)-\frac{i}{2} \Gamma_{n m^{\prime}}\left(\Delta_{2}\right)=\frac{\left|V_{n m^{\prime}}\right|^{2}}{\Delta_{2}+i \Gamma / 2} .
$$

The susceptibility tensor (2.3) describes an anisotropic and optically active medium despite the homogeneous population of the Zeeman sublevels. This is a direct consequence of the EIT effect. Anisotropy comes from the different $\Lambda$-type transitions activated by differently polarized probe modes. This effect has a similar physical nature to various optical anisotropy effects associated with the presence of the strong coupling field, see Refs. [23-25].

The scattering process in a medium is conveniently described by the scattering tensor formalism. This tensor is responsible for frequency- and polarization-dependent transformation of an incident electromagnetic plane wave as a result of its scattering on an isolated atom. Under conditions of EIT control for the mode incident on atom at frequency $\omega_{2}$, the scattering tensor is given by

$$
\begin{aligned}
\hat{\alpha}_{p q}^{\left(m^{\prime \prime} m\right)}\left(\Delta_{2}\right) \equiv & \alpha_{p q}^{\left(m^{\prime \prime} m\right)}\left(\Delta_{2}\right)\left|m^{\prime \prime}\right\rangle\langle m| \\
= & -\sum_{m^{\prime}(n), n} \frac{1}{\hbar} \frac{\left(d_{p}\right)_{m^{\prime \prime} n}\left(d_{q}\right)_{n m}}{\Delta_{2}+i \Gamma / 2}\left|m^{\prime \prime}\right\rangle\langle m| \\
& \times\left\{1-\frac{\left|V_{n m^{\prime}}\right|^{2}}{\Delta_{2}+i \Gamma / 2} \frac{1}{\Delta_{1}-\Delta_{2}+\Sigma_{n m^{\prime}}\left(\Delta_{2}\right)}\right\},
\end{aligned}
$$

which determines the amplitude of the outgoing wave for either the elastic or inelastic scattering channel accompanied by transition of the atom from the state $|m\rangle \equiv|F m\rangle$ with $F$ $=1$ to the state $\left|m^{\prime \prime}\right\rangle \equiv\left|F m^{\prime \prime}\right\rangle$ with $\mathrm{F}=1$ (Rayleigh channel) or $F=2$ (inelastic Raman channel). The scattering tensor should obey the following important identity

$$
\begin{aligned}
& \int d \Omega \sum_{m^{\prime \prime} \varepsilon^{\prime}} \frac{\omega_{2}\left(\omega_{2}+\omega_{m m^{\prime \prime}}\right)^{3}}{c^{4}}\left|\alpha_{p q}^{\left(m^{\prime \prime} m\right)}\left(\Delta_{2}\right)\left(\varepsilon^{\prime *}\right)^{p} \varepsilon^{q}\right|^{2} \\
& =\frac{4 \pi \omega_{2}}{c} \operatorname{Im}\left[\alpha_{p q}^{(m m)}\left(\Delta_{2}\right)\left(\varepsilon^{*}\right)^{p} \varepsilon^{q}\right],
\end{aligned}
$$

which is an optical theorem reflecting the unitary property of the scattering process. Here $\varepsilon^{q}$ and $\left(\varepsilon^{\prime *}\right)^{p}$ are the contravariant circular components of the polarization vectors for the incident photon and of the complex conjugated polarization vector, respectively, for the scattered photon. The sum is extended over all possible scattering channels and all possible output polarizations. After integration over the full scattering angle, the left-hand side reproduces the total scattering cross section.

Because of their similar physical nature, the expressions for the susceptibility tensor (2.3), and for the scattering tensor (2.5) are visualized in similar form. The important difference is that in the expression (2.5), an arbitrary reference frame can be assumed and any dipole-type transitions $|m\rangle$ $\rightarrow|n\rangle$ can be initiated. At the same time, each of the upper states $|n\rangle$ is coupled by the strong field with a certain selected ground state $\left|m^{\prime}\right\rangle$ that is indicated in the sum as $m^{\prime}=m^{\prime}(n)$. This prohibits the light scattering for the resonance mode when $\Delta_{2}=\Delta_{1}$ but opens the scattering channel for any nonvanishing detuning. Moreover, under the EIT effect it becomes quite important which scattering channel (elastic Rayleigh or inelastic Raman) the probe photon occupies as it is scattered away from its original forward propagation. An 
elastically scattered photon still undergoes coherent coupling and should emerge from the sample with a relatively long delay. In contrast, in the Raman channel the photon freely propagates through the sample with nearly the (vacuum) speed of light, because the medium is ideally transparent in this case.

\section{The Green's function}

Since the probe or scattered beam can propagate in any direction, its free-path transformation can be properly described by the Green's function formalism. For the transmitted or elastically scattered light the Green's function can be expressed in the form of phase integrals

$$
\begin{aligned}
D_{q_{1}}{ }^{q_{2}}\left(\mathbf{r}_{1}, \mathbf{r}_{2}, \omega_{2}\right)= & -\frac{\hbar}{\left|\mathbf{r}_{1}-\mathbf{r}_{2}\right|} e^{i \phi_{0}\left(\mathbf{r}_{1}, \mathbf{r}_{2}\right)+i k_{2}\left|\mathbf{r}_{1}-\mathbf{r}_{2}\right|} \\
& \times\left[\cos \left(\phi\left(\mathbf{r}_{1}, \mathbf{r}_{2}\right)\right) \delta_{q_{1}}{ }^{q_{2}}\right. \\
& \left.+i \sin \left[\phi\left(\mathbf{r}_{1}, \mathbf{r}_{2}\right)\right](\vec{n} \hat{\vec{\sigma}})_{q_{1}}{ }^{q_{2}}\right]
\end{aligned}
$$

Here $k_{2}=\omega_{2} / c$ and $\vec{n}=\vec{n}\left(\Delta_{2}\right)$ is the "unit" symbolic vector, the components of which are defined below.

The parameters of the phase integrals can be expressed by the major components of the susceptibility tensor, which are defined by Eq. (2.3) and related to the probe beam either copropagating in the forward direction or perpendicular to the coupling beam. In this sense, expression (2.7) gives a radiation zone asymptote for the retarded-type Green's function and the polarization indices $q_{1}, q_{2}$ are defined in the reference frame naturally linked with the ray direction, such that $z \| \mathbf{r}_{1}-\mathbf{r}_{2}$. In this frame the Green's function has a nondiagonal form in a natural basis of local circular polarizations with $q_{1,2}= \pm 1$ (left-hand/right-hand).

The phase integrals are given by

$$
\begin{gathered}
\phi_{0}\left(\mathbf{r}_{1}, \mathbf{r}_{2}\right)=\frac{2 \pi \omega_{2}}{c} \int_{\mathbf{r}_{2}}^{\mathbf{r}_{1}} \chi_{0}\left(\mathbf{r}, \Delta_{2}\right) d s \\
\phi\left(\mathbf{r}_{1}, \mathbf{r}_{2}\right)=\frac{2 \pi \omega_{2}}{c} \int_{\mathbf{r}_{2}}^{\mathbf{r}_{1}} \chi\left(\mathbf{r}, \Delta_{2}\right) d s
\end{gathered}
$$

and evaluated along a ray from point $\mathbf{r}_{2}$ to point $\mathbf{r}_{1}$. The integrand $\chi_{0}\left(\mathbf{r}, \Delta_{2}\right)$ is expressed in terms of major-axes components of the susceptibility tensor as

$$
\begin{aligned}
\chi_{0}\left(\mathbf{r}, \Delta_{2}\right)= & \frac{1+\cos ^{2} \beta}{4}\left[\chi_{-1}^{-1}\left(\mathbf{r}, \Delta_{2}\right)+\chi_{+1}^{+1}\left(\mathbf{r}, \Delta_{2}\right)\right] \\
& +\frac{\sin ^{2} \beta}{2} \chi_{0}^{0}\left(\mathbf{r}, \Delta_{2}\right)
\end{aligned}
$$

where $\beta$ is the polar angle between the ray and the coupling beam directions. The function $\chi\left(\mathbf{r}, \Delta_{2}\right)$ is given by

$$
\chi\left(\mathbf{r}, \Delta_{2}\right)=\left[\sum_{\mathrm{i}=1}^{3} \chi_{\mathrm{i}}^{2}\left(\mathbf{r}, \Delta_{2}\right)\right]^{1 / 2}
$$

where

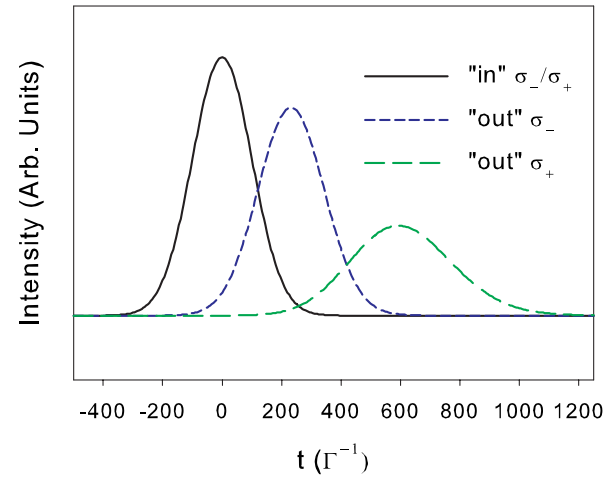

FIG. 2. (Color online) Intensity profiles for light pulses transmitted through a sample of ${ }^{87} \mathrm{Rb}$ atoms via the EIT channels. The coupling field is applied on the empty $F=2 \leftrightarrow F^{\prime}=1$ transition with right-handed circular polarization and the probe field, exciting the atoms on the $F=1 \rightarrow F^{\prime}=1$ transition, is either left-handed polarized (blue, short-dash curve) or right-handed polarized (green, longer dash curve). The black curve indicates the original Gaussian-type pulse with a duration of $\tau_{p}=100 \Gamma^{-1}$.

$$
\begin{aligned}
\chi_{1}\left(\mathbf{r}, \Delta_{2}\right)= & \frac{\sin ^{2} \beta}{2} \cos \gamma\left\{\frac{1}{2}\left[\chi_{-1}^{-1}\left(\mathbf{r}, \Delta_{2}\right)+\chi_{+1}^{+1}\left(\mathbf{r}, \Delta_{2}\right)\right]\right. \\
& \left.-\chi_{0}^{0}\left(\mathbf{r}, \Delta_{2}\right)\right\}, \\
\chi_{2}\left(\mathbf{r}, \Delta_{2}\right)= & \frac{\sin ^{2} \beta}{2} \sin \gamma\left\{\frac{1}{2}\left[\chi_{-1}^{-1}\left(\mathbf{r}, \Delta_{2}\right)+\chi_{+1}^{+1}\left(\mathbf{r}, \Delta_{2}\right)\right]\right. \\
& \left.-\chi_{0}^{0}\left(\mathbf{r}, \Delta_{2}\right)\right\}, \\
\chi_{3}\left(\mathbf{r}, \Delta_{2}\right)= & \frac{\cos \beta}{2}\left[\chi_{+1}^{+1}\left(\mathbf{r}, \Delta_{2}\right)-\chi_{-1}^{-1}\left(\mathbf{r}, \Delta_{2}\right)\right] .
\end{aligned}
$$

Here $\gamma$ is an azimuthal angle indicating the uncertainty in directions of a pair of orthogonal axes, fixing the reference frame in the plane transverse to the ray. The "unit" symbolic vector $\vec{n}$ is defined by its components as

$$
n_{\mathrm{i}}=n_{\mathrm{i}}\left(\Delta_{2}\right)=\frac{\chi_{\mathrm{i}}\left(\mathbf{r}, \Delta_{2}\right)}{\chi\left(\mathbf{r}, \Delta_{2}\right)},
$$

and is independent of $\mathbf{r}$. It is described in the general case by the set of complex components $\left(n_{1}, n_{2}, n_{3}\right)$.

\section{RESULTS}

\section{A. The forward transmitted pulse}

The graphs in Figs. 2 and 3 show how the original pulse profile is modified after propagation through the sample in an EIT channel. The calculations were done for two different pulse shapes, these being Gaussian-type (Fig. 2) and rectangular-type (Fig. 3), with pulse widths of $\tau_{p}=100 \Gamma^{-1}$. In our numerical simulations we considered as an example ${ }^{87} \mathrm{Rb}$ atoms, when the coupling field and the probe light excitations were respectively applied at $F=2 \leftrightarrow F^{\prime}=1$ (empty) 


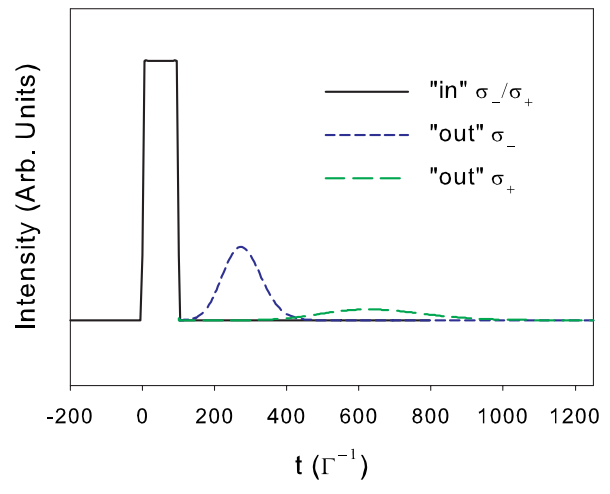

FIG. 3. (Color online) Same as in Fig. 2 but for a rectangular input pulse profile.

and $F=1 \rightarrow F^{\prime}=1$ (equally populated) hyperfine transitions, as shown in Fig. 1. The coupling field is always right-hand circularly polarized and the probe field can have varying polarization, being either left- or right-hand circularly polarized. The calculations were based on attainable parameters of an ultracold atomic cloud confined in a magneto-optic trap. For a Gaussian atom distribution in the trap, the weak-field optical depth, on resonance and through the center of the trap, is given by $b_{0}=\sqrt{2 \pi} n_{0} \sigma_{0} r_{0}$, where $n_{0}$ is the peak density and $\sigma_{0}$ is the normal (not modified by the EIT effect) resonance cross section, and $r_{0}$ is the radius of the cloud. The dependencies shown in Figs. 2 and 3 reproduce the pulse transformation after its passing through such an atomic sample with the size $r_{0}=0.25 \mathrm{~cm}$ and peak optical depth $b_{0}$ $=50$. All the parameters are in accordance with the condition that the delay time $\tau_{d}$ is longer than the duration $\tau_{p}$ of the original pulse. The inequality $\tau_{d}>\tau_{p}$ can be controlled by appropriate choice of the Rabi frequency for the coupling field $\Omega_{c}=2\left|V_{n m^{\prime}}\right|$, which we defined with respect to the $m^{\prime}$ $=\{F=2, M=-1\} \leftrightarrow n=\left\{F^{\prime}=1, M^{\prime}=0\right\}$ hyperfine Zeeman transition. For the dependencies shown in Figs. 2 and 3, the Rabi frequency is given by $\Omega_{c}=0.4 \Gamma$.

For the case of a Gaussian profile the output pulse preserves the original shape but becomes more extended in time. The highest fidelity channel for reproduction of the original pulse is obtained when the coupling and probe fields are in orthogonal polarization states (blue curve in Fig. 2). The loss of the pulse energy is only $10 \%$ for this case, but for the pulse transmitted through the sample in the right-handed polarization channel there is up to $40 \%$ energy loss. This is a clear manifestation of the scattering process, which cannot be ignored, as we argued by simple estimations in Sec. II A.

The modification of the pulse profile manifests itself even more dramatically if the probe pulse has a rectangular shape. Due to dispersion the original pulse shape is completely transformed in the extended medium to a Gaussian form and the losses of energy are more significant for this case. That effect is shown by the calculations of the outgoing pulse profile presented in Fig. 3 for two different polarization channels. The calculations were done for the initial parameters similar to those used in the calculation of the dependencies for Fig. 2. For the outgoing pulses shown in Fig. 3 the losses are up to $60 \%$ for the left-handed and up to $80 \%$ for the right-handed polarization channels.

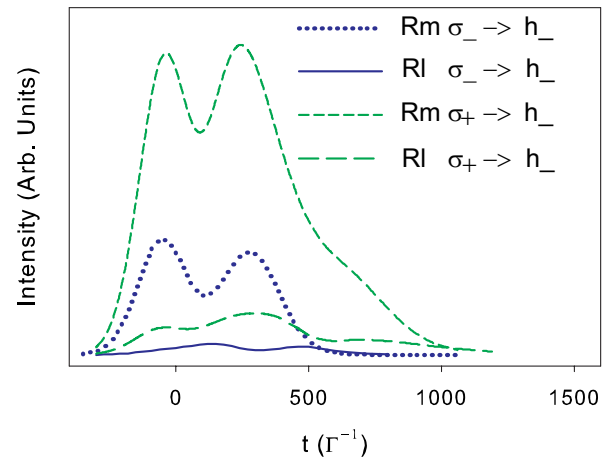

FIG. 4. (Color online) The intensity profiles for the portion of the light pulse scattered at $90^{\circ}$ to the direction of the incident pulse. The curves represent the Rayleigh (Rl) channel $\left(F=1 \rightarrow F^{\prime}=1\right.$ $\rightarrow F=1)$ and the Raman (Rm) channel $\left(F=1 \rightarrow F^{\prime}=1 \rightarrow F=2\right)$, with the input polarization state, and polarization channel of the emerging light as indicated in the caption. In all cases, the observation channel corresponds to detection of light with left-hand helicity $\left(h_{-}\right)$.

Let us comment on the properties of the transmitted pulse, as displayed in Figs. 2 and 3, in the context of our previous discussion given in Sec. II A. As one can see from the figures, the behavior of the wave packet, forwardly propagating through the sample, critically depends on its intrinsic spectral properties. For the smoothed spectral expansion, and particularly for the Gaussian-type spectral distribution, the energy losses are basically determined by a near resonance overlap of the wave packet spectral components with the EITmodified absorption spectral profile. As far as this overlap can be effectively minimized by optimal choice of the pulse spectral extension, as was described in Sec. II A, the level of losses can be also minimized. As follows from our calculations, this takes place for the left-handed circular polarized probe light, and the best data for the transmitted pulse are reproduced in Fig. 2. Such an optimization would be not possible to do for a pulse profile having a rectangular shape. Then there is a non-negligible and even significant contribution into the integral absorption coefficient, which is determined by the spectral overlap of the Lorentzian wings of the absorption spectrum, with far spectral components responsible for the step-type shape of the temporal pulse profile. The optimal conditions formulated in Sec. II A can never be ideally fulfilled for this case. It seems to us also important that for a rectangular pulse such far off-resonant spectral components can be partly transmitted through the sample via a normal (not EIT) transparent domain. This results in sharp transient processes in the time behavior for the transmitted pulse at the beginning and final stages, which for the sake of simplicity are not shown in the graphs of Fig. 3.

\section{B. The scattered pulse}

The plots in Fig. 4 reproduce the time dependence of instantaneous intensity for the fractions of the light pulse, originally incoming with Gaussian profile shown in Fig. 2, and scattered by the sample in a direction orthogonal to the incident probe beam. Note that we designate the polarization 
of the scattered light in terms of its helicity, which is a natural choice when considering the radiation propagating toward a detector. In Fig. 4, the curves indicate our results for the Rayleigh scattering channel $\left(F=1 \rightarrow F^{\prime}=1 \rightarrow F=1\right)$ and the Raman channel $\left(F=1 \rightarrow F^{\prime}=1 \rightarrow F=2\right)$. Let us recall here that for the sake of brevity we do not distinguish between elastic Raman and Rayleigh channels. The calculational parameters were chosen the same as for the dependencies of Figs. 2 and 3. The solid and dotted blue curves illustrate the portion of the scattered light pulse incoming with left-handed circular polarization $\left(\sigma_{-}\right)$and emerging the sample in the left-hand helicity polarization $\left(h_{-}\right)$. In turn, the short-dashed and long-dashed green curves illustrate the portion of the scattered light pulse incoming with right-handed polarization $\left(\sigma_{+}\right)$and emerging the sample again in the left-hand helicity polarization $\left(h_{-}\right)$. Thus, for the intensity profiles shown in Fig. 4, the outgoing light is always considered in the lefthand helicity polarization channel.

As follows from these dependencies, the scattered light emerges from the sample with a rather long delay in units of $\Gamma^{-1}$. This is an indication of the slow light phenomenon typically associated with the EIT effect, but for the scattered light. The portion of the input pulse, distorted in the scattering process, propagates through the sample faster and is more extended in time than the transmitted pulse. This can be explained by the spectral structure of the scattered pulse, which has a deficit of near-resonance photons for which the EIT mechanism works ideally. For the scattered light pulse leaving the sample via a Rayleigh channel, the group velocity is greater than for the transmitted incident pulse. The photons created in the Raman channel leave the sample with the speed of light in vасио because the medium is fully transparent for them. The spectral notch in the distribution of the scattered photons leads to the beating effect in the time behavior of the output light intensity, which is clearly seen for all the scattering channels presented in Fig. 4.

To show the difference between the usual incoherent scattering and scattering in the environment supporting the EIT effect, let us turn to the physical background of the process. If the medium is illuminated by a single-mode probe field, which is precisely in the resonance with the coupling field such that $\omega_{1}=\omega_{2}+\omega_{21}$, where $\omega_{21}$ is the hyperfine splitting in the diagram of Fig. 1, the entire atom-field system is described by the following density matrix

$$
\hat{\rho}=\frac{1}{3} \sum_{j=1,2}\left|\Psi_{j}\right\rangle\left\langle\Psi_{j}\right|+\ldots
$$

where dots denote the contribution of noncoupled state and the sum $j=1,2$ is extended over two $\Lambda$ schemes shown in Fig. 1. The wave functions contributing to the expansion (3.1) are given by

$$
\left.\left|\Psi_{j}\right\rangle=\left[\frac{\Omega_{p}^{(j)}}{\sqrt{\Omega_{p}^{(j) 2}+\Omega_{c}^{(j) 2}}}|1\rangle_{j}-\frac{\Omega_{c}^{(j)}}{\sqrt{\Omega_{p}^{(j) 2}+\Omega_{c}^{(j) 2}}}|2\rangle_{j}\right] \times \mid \text { Field }\right\rangle
$$

where $|1\rangle_{j}$ and $|2\rangle_{j}$ are, respectively, the left and right atomic spin states coupled with the $j$ th $\Lambda$-type excitation channel, and $\Omega_{c}^{(j)}$ and $\Omega_{p}^{(j)}$ are the Rabi frequencies respectively for the coupling and for the probe fields. For the sake of simplicity we presume both the Rabi frequencies to be real parameters. It is a crucial point of the EIT effect that despite the fact that even though the atomic substate given in the square brackets of (3.2) is not an eigenstate of the atomic Hamiltonian, the full wave function $\left|\Psi_{j}\right\rangle$ is an eigenstate of the entire atom-field system if the field is in coherent state. Thus, under conditions of perfect $\Lambda$ resonance the system is not able to scatter the light.

However if the probe mode is not in exact two-photon resonance the situation becomes more complicated. In the single-atom case and as a first approximation there will be coherent beats with exchange of a photon between coupling and probe modes of the field, which are initiated by lowfrequency phase oscillation of the atomic spin coherence. In a macroscopic system these beats are collectivized and transformed to a polariton wave packet created in the sample by a pulse of the probe field enveloped by a set of near resonance spectral modes. For a short distance the polariton wave propagates in the forward direction, but it will be scattered at long distances if the medium is optically extended. However, the probe field, even being scattered via Rayleigh channels, remains in a coherent state and can be also enveloped by the modes inside the EIT spectral window. Thus, the polariton wave does not disappear as a result of Rayleigh scattering but transforms to a diffuse coherent mode. The quantum nature of such a diffuse polariton can be verified if the control field is switched off and switched on again with delay. Then the propagation of the polariton wave in the forward direction and via the Rayleigh scattering channel will be stopped and be regenerated again with a time delay. Thus, the scattered light, as well as the transmitted light, can be stored in the medium and recovered on demand. That is a unique property of the light propagation under EIT conditions.

\section{The coherent backscattering process}

A clear indicator of the coherence in the Rayleigh scattering channel is the appearance of the coherent backscattering (CBS) process. This effect is a typical example of a "which path" interference fashioned by a disordered medium. For a portion of a light wave emerging the sample in the backward direction there is constructive, or for some special cases, destructive interference [26] between the scattering amplitudes along any multiple scattering chain. The various manifestations of the CBS phenomenon in ultracold atomic systems [27-29], and under different physical conditions, has been the subject of many discussions during the last decade (see Kupriyanov et al. for a review [30]). Some future prospects in this field have been summarized by Havey and Kupriyanov [31]. In the context of light scattering in an atomic environment supporting the EIT effect, the CBS process can be interpreted as interference between two unknown reciprocal paths for the diffuse polaritonic wave propagating inside the sample via Rayleigh scattering as shown schematically in Fig. 5. The specific nature of the EIT resonance essentially modifies the time behavior of the interference component of the outgoing wave packet in comparison with how it is usu- 


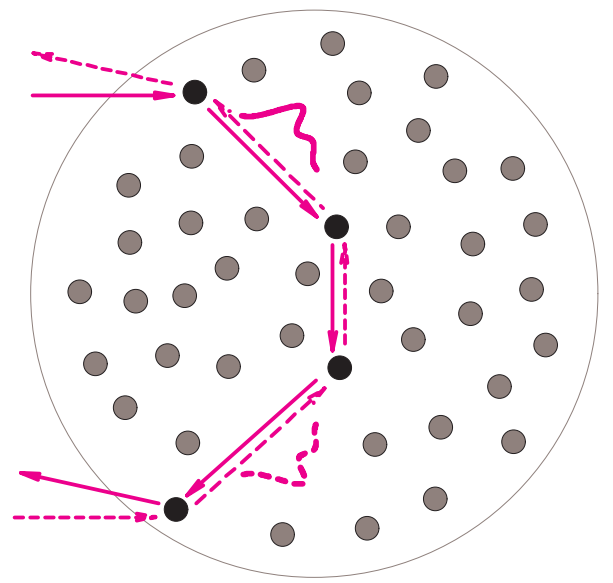

FIG. 5. (Color online) A schematic diagram explaining the coherent backscattering phenomenon. The polaritonic waves scattered in the medium follow pairs of reciprocal scattering paths indicated by the solid and dashed traces. The emergent electromagnetic field in the backward direction can show interference between the propagating modes following the two paths.

ally observed for a pulse-type excitation of an opaque atomic medium.

For the scattering process developing in the standard conditions of elastic scattering and for a rather long excitation probe pulse, the "ladder" (noninterference) and the "crossed" (interference) components have an approximately similar dependence on time. The important difference in their time behavior is mainly observable in the transient stages of the excitation process (see Ref. [30]). Inside the pulse the relation between the instantaneous magnitudes of these components, usually expressed in terms of the enhancement factor for the CBS output intensity, has typically no time dependence. The enhancement factor approaches a stable and constant value inside the pulse. This value reproduces the enhancement factor for the steady-state regime in conditions of a single-mode excitation of the atomic sample with a resonant probe light. A crucial contrast for the CBS process observable in an environment of the EIT effect is that the resonant carrier mode of the light pulse cannot be scattered and the spectral profile for the pulse fraction, deflected by the medium from its original forward propagation, always has a narrow gap near this mode. That results in a quite unusual time behavior for the interference overlap of two reciprocal fragments of the backscattered wave packet.

The dependencies of Figs. 6 and 7 illustrate the temporal behavior of the instantaneous intensity for the fraction of the probe pulse scattered in the backward direction via the Rayleigh channels $\sigma_{-} \rightarrow h_{-}$and $\sigma_{+} \rightarrow h_{-}$. The outgoing intensity is mainly contributed by the ladder-type terms such that the interference contribution, shown in the magnified bottom parts of the graphs, gives actually only a few percent of the total intensity. For convenience in these figures, the gray curves indicate the time profiles for the incoming and transmitted pulses. Let us point out that these profiles slightly differ from those which are shown in Figs. 2 and 4 because the aperture of the probe beams are selected differently for the two calculations. In Fig. 2 the cross section of the beam

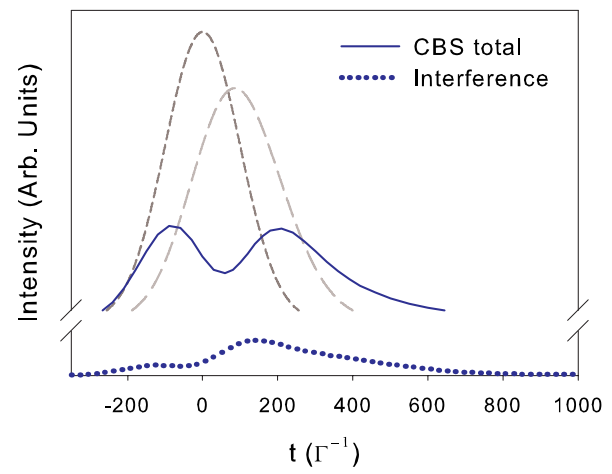

FIG. 6. (Color online) The temporal profiles for the fraction of the light pulse scattered in the backward direction for the $\sigma_{-} \rightarrow h_{-}$ Rayleigh scattering channel. The blue solid curve is the total intensity profile and the dotted blue curve is the interference contribution. The short- and long-dashed gray curves, respectively, indicate the input and the transmitted pulses. For these graphs the ordinate only qualitatively reproduces the relative magnitudes of the pulse intensities.

was less than the cross-sectional area of the atomic cloud, which is a natural requirement for measurements in slow or stopped light experiments. Alternatively, for observing the CBS effect from all atoms of the ensemble, it is more natural to make the cross section of the probe beam bigger than the cross-sectional area of the atomic cloud. The important feature of the dependencies displayed in Figs. 6 and 7 is that the crossed-type terms lead to an oscillating interference enhancement near the midpoint of the backscattered pulse profile.

The time behavior of the interference effect can be properly described in terms of the enhancement factor

$$
\eta(t)=\frac{I_{S}(t)+I_{L}(t)+I_{C}(t)}{I_{S}(t)+I_{L}(t)},
$$

where $I_{S}(t)+I_{L}(t)$ is the contribution of single scattering and of the ladder terms in multiple scattering and $I_{C}(t)$ is the contribution of the crossed terms. The dependence of $\eta(t)$ on time is illustrated in the graphs of Fig. 8. It is an intriguing consequence of the calculation results that the behavior of the enhancement factor is generally quite complicated be-

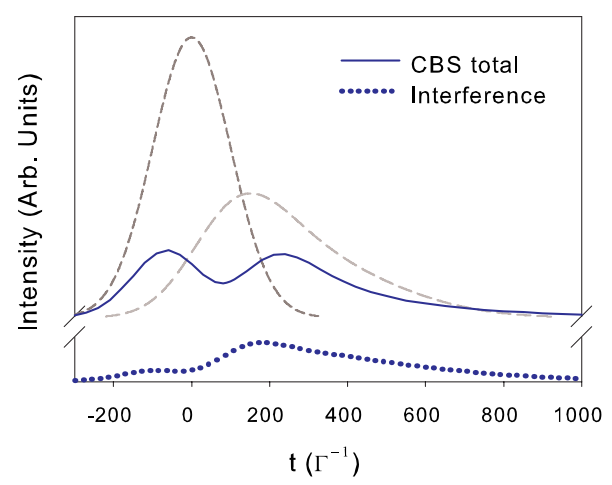

FIG. 7. (Color online) Same as in Fig. 6 but for the $\sigma_{+} \rightarrow h_{-}$ Rayleigh scattering channel. 


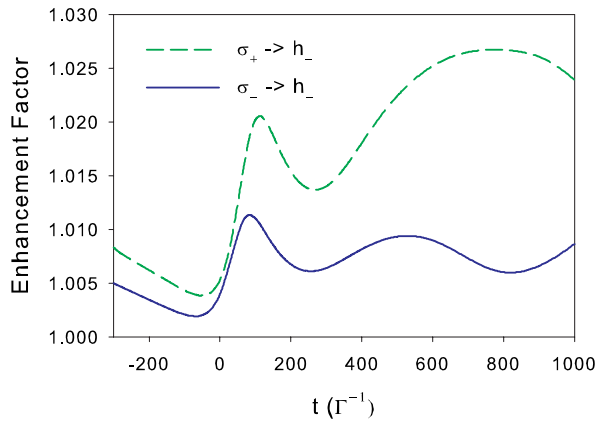

FIG. 8. (Color online) The enhancement factor for $\sigma_{+} \rightarrow h_{-}$ (green dashed curve) and $\sigma_{-} \rightarrow h_{-}$(blue solid curve) scattering channels.

cause of the nontrivial manifestation of different spectral components of the input pulse in formation of the backscattered fraction of the outgoing pulse in different scattering orders. The most important seems an oscillating enhancement near the midpoint of the scattered pulse, which corresponds with the polaritonic wave packet overlap inside the medium.

\section{CONCLUSIONS}

In summary, we have made a theoretical and calculational investigation of the Rayleigh and Raman scattered light modes in a medium configured to demonstrate electromagnetically induced transparency in the coherently forward scattered light. In particular, for an anisotropic and inhomogeneous sample of ultracold atoms we have examined the time evolution of the forward scattered light, the diffusely (multiply) scattered light in a characteristic right-angle fluo- rescence geometry, and in the backscattering configuration used to study weak localization of light in ultracold atomic gases. The intensity of the forward scattered light is modified by both Rayleigh and Raman scattering of Fourier components of the incident pulse which lie outside the EIT transmission window. In particular, the intensity of a light pulse regenerated in a typical "stopped-light" experiment is significantly modified by the loss of these components, which in part maintain their coherence with respect to the incident light. In addition, these components produce complex time dependence in both the fluorescence and in the coherent backscattering geometries. The interferences responsible for the coherent backscattering enhancement are a clear indicator of the coherence of the scattering processes. We emphasize the fundamental conclusion that light scattered into the Rayleigh modes maintains its coherence with respect to the original incident pulse. This is manifested by interferometric enhancement of the backward scattered intensity from the sample. This in turn implies that, along with the polariton associated with the forward scattered light, a diffuse and coherently related polariton coexists within the volume of the ultracold atomic sample. We close by noting that a deeper understanding of the mesoscopic properties of the diffusive excitation is clearly required, and this report represents a first step in this direction. Further theoretical and experimental research into this area is underway.

\section{ACKNOWLEDGMENTS}

We appreciate the financial support of the National Science Foundation (NSF-PHY-0355024), the Russian Foundation for Basic Research (RFBR-05-02-16172-a), INTAS (Project ID: 7904), and the North Atlantic Treaty Organization (PST-CLG-978468). D.V.K. would like to acknowledge financial support from the Delzell Foundation, Inc.
[1] Stephen E. Harris, Phys. Today 50, 36 (1997).

[2] M. D. Lukin and A. Imamoglu, Nature (London) 413, 273 (2001).

[3] M. D. Lukin, Rev. Mod. Phys. 75, 457 (2003).

[4] Michael Fleischhauer, Atac Imamoglu, and Jonathan P. Marangos, Rev. Mod. Phys. 77, 633 (2005).

[5] Harold J. Metcalf and Peter van der Straten, Laser Cooling and Trapping (Springer, New York, 1999).

[6] L. V. Hau, S. E. Harris, Z. Dutton, and C. H. Behroozi, Nature (London) 397, 594 (1999).

[7] M. Fleischhauer and M. D. Lukin, Phys. Rev. A 65, 022314 (2002)

[8] Danielle A. Braje, Vlatko Balic, Sunil Goda, G. Y. Yin, and S. E. Harris, Phys. Rev. Lett. 93, 183601 (2004).

[9] Danielle A. Braje, Vlatko Balic, G. Y. Yin, and S. E. Harris, Phys. Rev. A 68, 041801(R) (2003).

[10] Chien Liu, Zachary Dutton, Cyrus H. Behroozi, and Lene Vestergaard Hau, Nature (London) 409, 490 (2001).

[11] Min Yan, Edward G. Rickey, and Yifu Zhu, Phys. Rev. A 64, 041801(R) (2001).

[12] D. Budker, D. F. Kimball, S. M. Rochester, and V. V. Yash- chuk, Phys. Rev. Lett. 83, 1767 (1999).

[13] M. M. Kash, V. A. Sautenkov, A. S. Zibrov, L. Hollberg, G. R. Welch, M. D. Lukin, Yu. Rostovtsev, E. S. Fry, and M. O. Scully, Phys. Rev. Lett. 82, 5229 (1999).

[14] A. B. Matsko, I. Novikova, M. O. Scully, and G. R. Welch, Phys. Rev. Lett. 87, 133601 (2001).

[15] Yanhong Xiao, Irina Novikova, David F. Phillips, and Ronald L. Walsworth, Phys. Rev. Lett. 96, 043601 (2006).

[16] T. Chanelière, D. N. Matsukevich, S. D. Jenkins, S.-Y. Lan, T. A. B. Kennedy, and A. Kuzmich, Nature (London) 438, 833 (2005).

[17] J. P. Marangos, J. Mod. Opt. 45, 471 (1998).

[18] E. Arimondo, in Progress in Optics, edited by E. Wolf (Elsevier Science, Amsterdam, 1996), Vol. XXXV, p. 257.

[19] Marlan O. Scully and M. Suhail Zubairy, Quantum Optics (Cambridge University Press, Cambridge, 1997).

[20] M. J. Collett and C. W. Gardiner, Phys. Rev. A 30, 1386 (1984).

[21] D. A. Varshalovich, A. N. Maskalev, and V. K. Khersonskii, Quantum Theory of Angular Momentum (World Scientific, Singapore, 1988). 
[22] It is important to recognize that in this complex basis set the contravariant basis vectors are given by $\mathbf{e}^{q}=\mathbf{e}_{q}^{*}$ and the metric tensor has nondiagonal form, $\left(\mathbf{e}_{q}\right)_{p}=(-)^{q} \delta_{q-p}$, such that a right-hand polarization vector with $q=+1$ has negative covariant projection with $p=-1$.

[23] D. McGloin, M. H. Dunn, and D. J. Fulton, Phys. Rev. A 62, 053802 (2000).

[24] D. Cho, J. M. Choi, J. M. Kim, Q. Han Park, Phys. Rev. A 72 , 023821 (2005).

[25] V. A. Sautenkov, Y. V. Rostovtsev, H. Chen, P. Hsu, G. S. Agarwal, and M. O. Scully, Phys. Rev. Lett. 94, 233601 (2005).

[26] D. V. Kupriyanov, I. M. Sokolov, and M. D. Havey, Opt. Commun. 243, 165 (2004).

[27] G. Labeyrie, F. deTomasi, J-C. Bernard, C. A. Müller, C. Miniatura, and R. Kaiser, Phys. Rev. Lett. 83, 5266 (1999). See also, G. Labeyrie, C. A. Müller, D. S. Wiersma, Ch. Miniatura, and R. Kaiser, J. Opt. B: Quantum Semiclassical Opt. 2, 672 (2000).

[28] Y. Bidel, B. Klappauf, J. C. Bernard, D. Delande, G. Labeyrie, C. Miniatura, D. Wilkowski, and R. Kaiser, Phys. Rev. Lett. 88, 203902-1 (2002).

[29] D. V. Kupriyanov, I. M. Sokolov, P. Kulatunga, C. I. Sukenik, and M. D. Havey, Phys. Rev. A 67, 013814 (2003); D. V. Kupriyanov, I. M. Sokolov, N. V. Larionov, P. Kulatunga, C. I. Sukenik, S. Balik, and M. D. Havey, Phys. Rev. A 69, 033801 (2004).

[30] D. V. Kupriyanov, I. M. Sokolov, C. I. Sukenik, and M. D. Havey, Laser Phys. Lett. 3, 223 (2006).

[31] Mark D. Havey and Dmitriy V. Kupriyanov, Phys. Scr. 72, C30 (2005) 